\title{
Synthesis and Characterization of an Efficient New Liquid Laser Dye Material - Chalcone (DMAPPP)
}

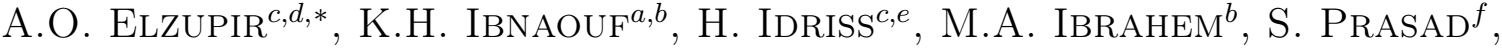 \\ M.A. Alrajhi ${ }^{a, c}$, M.S. AlSalhi ${ }^{f, g}$ AND A.S. AlaAmer ${ }^{a, c}$ \\ ${ }^{a}$ Al Imam Mohammad Ibn Saud Islamic University (IMSIU), College of Science, Physics Department, \\ P.O. Box 90950, Riyadh, 11623, Saudi Arabia \\ ${ }^{b}$ Alneelain University, School of Physics, Faculty of Science and Technology, \\ P.O. Box 12702, Khartoum, 11121 Sudan \\ ${ }^{c}$ Al Imam Mohammad Ibn Saud Islamic University (IMSIU), College of Science, \\ Committee on Radiation and Environmental Pollution Protection, Riyadh, KSA \\ ${ }^{d}$ Ministry of Higher Education, Central Laboratory, Khartoum, Sudan \\ ${ }^{e}$ Ministries of Higher Education and Scientific Research, Sudan Atomic Energy Commission, Khartoum, Sudan \\ ${ }^{f}$ KSU Research Chair on Laser Diagnosis of Cancers, College of Science, King Saud University, \\ Riyadh, 11451 Saudi Arabia \\ ${ }^{g}$ Department of Physics and Astronomy, College of Science, Riyadh, 11451 Saudi Arabia \\ (Received May 17, 2017; revised version November 26, 2017; in final form December 17, 2017) \\ This paper comprises the synthesis and characterization of 3-(4-(dimethylamino)phenyl)-1-phenyl-(2E)- \\ propen-1-one (DMAPPP) and its application as a new laser medium. The absorption and fluorescence spectra of \\ DMAPPP under different solvents and concentrations have been investigated. The amplified spontaneous emission \\ performance of DMAPPP under various concentrations, organic solvents and pump pulse energies of Nd:YAG laser \\ $(355 \mathrm{~nm})$ was also studied. The amplified spontaneous emission spectra of DMAPPP in solution were compared \\ with a conventional laser dye of coumarin 503, under the same identical conditions. The gain and the fluorescence \\ quantum yield of DMAPPP were determined. The most important features are: (1) DMAPPP has an excellent \\ photochemical stability, (2) the amplified spontaneous emission from the DMAPPP was tuned in the wavelength \\ region between 515 and $548 \mathrm{~nm}$. This could be the first detailed paper on laser properties of DMAPPP.
}

DOI: 10.12693/APhysPolA.133.121

PACS/topics: chalcone, DMAPPP, solvents effect, fluorescence, ASE

\section{Introduction}

Chalcones represents an important family of natural as well as synthetic organic compound. These chalcones had stimulated wide research interest owing to their unique structural feature, which has a carbonyl functional group in conjugation with carbon-carbon double bond, known as $\alpha, \beta$-unsaturated keto group or enone function. This structural arrangement gives rise to numerous chemical, physical, photophysical and biological properties [1-8].

First report on chalcones and related compounds as an optical material came before few decades by Kitaoka et al. [4]. In spite of it there are versatile chemical and physical properties, and there are very limited data describing the photochemical and physical properties of these compounds. Recent reports related cocoons are widely used for various optical applications including second harmonic generation materials in nonlinear optics, photorefractive polymers, holographic recording materials and fluorescent probes for sensing of metal ions [9-18]. How-

*corresponding author; e-mail: aminosman81@gmail.com ever, the photophysical properties of these chalcones such as solvent environment, temperature effect and quantum yield of fluorescence were not fully studied.

The revival of chalcones as optical materials are due to the following facts: (1) new optical materials are needed for developments in the field of technology of such organic electronics, organic photonics, (2) low cost, easy and green materials, (3) it can be used as biocompatible and non-toxic for the human being, and (4) high thermal stability. Thus, the photophysical properties of chalcones producing ASE is important.

In our previous work we have got amazing results of ASE from 1-(4-chlorophenyl)-3-(4- $N, N$ dimethyl amino phenyl)-2-propen-1-one (CPDAPP) [19]. In the present paper, the photophysical properties and amplified spontaneous emission (ASE) as well as the photochemical stability of DMAPPP in different solvents and concentrations are described. The ASE results were compared with a conventional laser dye coumarin 503.

\section{Experimental}

\subsection{Chemical synthesis and characterization}

The DMAPPP was synthesized using the condensation reaction between acetophenone $(0.01 \mathrm{~mol})$ and 4 dimethylaminobenzaldehyde $(0.01 \mathrm{~mol})$ in basic condition of sodium hydroxide $(1.0 \mathrm{~g})$, in ethanol $(50 \mathrm{ml})$. 
The vessel was left overnight under steering at room temperature and then the yield was recrystallized from ethanol. The crystal was washed with distilled water and left to dry (yield 94\%) [1]. The product was characterized by $\mathrm{H}^{1}-\mathrm{NMR}, \mathrm{C}^{13}-\mathrm{NMR}$, mass, and $\mathrm{UV}$ vis spectroscopy. ${ }^{\delta} \mathrm{H}:(400 \mathrm{MHz}, \mathrm{CDCl} 3, \mathrm{Me} 4 \mathrm{Si}), 3.05$ $(6 \mathrm{H}, \mathrm{s}, \mathrm{NMe} 2), 6.67(2 \mathrm{H}, \mathrm{d}, J=8.9 \mathrm{~Hz}), 7.32(1 \mathrm{H}, \mathrm{d}$, $J=15.5 \mathrm{~Hz}), 7.48(2 \mathrm{H}, \mathrm{t}, J=7.7 \mathrm{~Hz}), 7.52(3 \mathrm{H}, \mathrm{m}$, $\mathrm{Ph}), 7.76(1 \mathrm{H}, \mathrm{d}, J=15.5 \mathrm{~Hz}), 7.99(2 \mathrm{H}, \mathrm{d}, J=8.3 \mathrm{~Hz})$ ppm. ${ }^{\delta} \mathrm{C}: 40\left(\mathrm{~N}\left(\mathrm{CH}_{3}\right)_{2}\right) ; 112(\mathrm{C}=\mathrm{C}) ; 117(\mathrm{C}=\mathrm{C}) ; 122(\mathrm{Ph})$; $128.3(\mathrm{Ph}) ; 128.4(\mathrm{Ph}) ; 130.5(\mathrm{Ph}) ; 132(\mathrm{Ph}) ; 139.5(\mathrm{Ph})$; $146(\mathrm{Ph}) ; 152(\mathrm{Ph}) ; 190(\mathrm{C}=\mathrm{O}) . \quad M / z(\%): 251(\mathrm{M} \pm 100)$; $\lambda_{\max }$ (methanol 265, 420).

\subsection{Aspectral analysis}

DMAPPP was dissolved in different organic solvents( a spectroscopic grade with purity $99.8 \%$ ). The molecular structure of DMAPPP is given in Fig. 1a. The absorption and fluorescence spectra of DMAPPP in acetone were studied under a wide range of concentrations. The spectra of these solutions were measured using a small quartz cuvette with the dimensions $1 \times 1 \times 4 \mathrm{~cm}^{3}$ with an optical path length of $1 \mathrm{~cm}$.

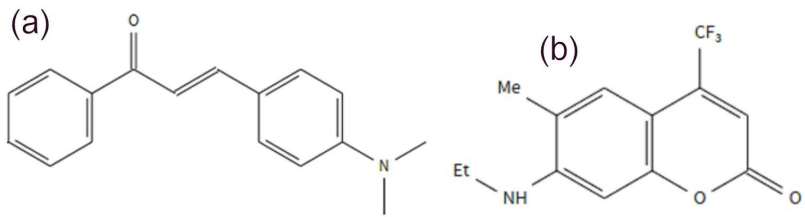

Fig. 1. Molecular structure of laser dyes: (a) DMAPPP, (b) coumarin 503.

The absorption spectra were taken using a PerkinElmer lambda 590 spectrophotometer over the range from 200 to $800 \mathrm{~nm}$ and the fluorescence spectra were recorded using a Perkin-Elmer LS55 spectrofluorometer in the range from 200 to $900 \mathrm{~nm}$, at room temperature. The excitation wavelength was $400 \mathrm{~nm}$.

The UV laser $(\lambda=355 \mathrm{~nm})$ was focused by a quartz plan-cylindrical lens with a focal length of $5 \mathrm{~cm}$. This was used to perform transverse excitation of the DMAPPP solution taken in a four-sided polished quartz cell, which was kept tilted to avoid feedback. See Ref. [20] for more details. Under suitable values of the pump power and concentrations of DMAPPP, the amplified spontaneous emission (ASE) was observed, coming out as a cone of light. This was collected by an optical fiber and fed into the $1 \mathrm{~mm}$ entrance slit of a spectrometer followed by a CCD camera (Ocean Optics) to obtain the spectral features of the amplified spontaneous emission. To avoid saturation of the CCD, we had used neutral density filters, as and when required. The ASE of the DMAPPP in solution was compared to that of coumarin 503 (see Fig. 1b) in ethanol at a concentration of $6.5 \mathrm{mM}$. It is important to note that these measurements were done under identical experimental conditions: only the dye cells were changed.

\section{Result and discussion}

\subsection{Spectral properties of DMAPPP}

DMAPPP was dissolved in different organic solvents with different dielectric constant. The concentration of these solutions was fixed at $6.5 \mathrm{mM}$. DMAPPP exhibits a large red shift in both absorption and emission spectra as the solvent polarity increased. It could be seen that the dielectric constant of the solvent plays an important role in the absorption and fluorescence bands. The influence of the solvents on the absorption and fluorescence spectra is shown in Table I.

\section{TABLE I}

The dielectric constant $\varepsilon$, spectral and ASE properties: $\lambda_{\max }[\mathrm{nm}]$ for absorption $(\mathrm{A})$, fluorescence $(\mathrm{F})$ and ASE; $\Phi_{F} ;$ ASE efficiency $\eta[\%]$ of DMAPPP in different solvents.

\begin{tabular}{l|c|c|c|c|c|c}
\hline \hline \multirow{2}{*}{ Solvent } & \multirow{2}{*}{$\varepsilon$} & \multicolumn{3}{|c|}{$\lambda_{\max }$} & \multirow{2}{*}{$\Phi_{F}$} & \multirow{2}{*}{$\eta$} \\
\cline { 3 - 6 } & & $\mathrm{A}$ & $\mathrm{F}$ & $\mathrm{ASE}$ & & \\
\hline benzene & 2.27 & 407 & 477 & - & 0.27 & - \\
toluene & 2.38 & 406 & 470 & - & 0.24 & - \\
chloroform & 4.81 & 417 & 510 & 523 & 0.66 & 22 \\
acetic acid & 6.15 & 423 & 517 & - & 0.05 & - \\
tetrahydrofuran & 7.58 & 404 & 506 & 515 & 0.96 & 46 \\
acetone & 20.7 & 407 & 519 & 532 & 0.88 & 31 \\
ethanol & 24.5 & 420 & 530 & 548 & 0.37 & 4 \\
methanol & 32.7 & 421 & 531 & - & 0.02 & - \\
dimethylformamide & 36.7 & 419 & 531 & 548 & 0.93 & 34 \\
acetonitrile & 37.5 & 410 & 532 & 547 & 0.66 & 9
\end{tabular}

In our previous communication we have reported that the intensity of the fluorescence decreases significantly as the concentration of the DMAPPP increased [19]. This may be due to the fact that two molecules are combined due to the dipole-dipole interaction in the excited states. The optical density of the absoption increased with an increase of concentration, although the shape of the absorption spectra remained the same irrespective of the concentration.

\subsubsection{The Stokes shift}

DMAPPP was dissolved in various organic solvents with different dielectric constants. The concentration of these solutions was fixed at $0.65 \mathrm{mM}$. The changes in the absorption and fluorescence spectra were observed. Figure 2 shows the variation of the Stokes shift as a function of the dipole factor of the solvent, as defined by Lippert and Mataga et al. [21, 22]. It can be seen that DMAPPP in the solution undergoes significant changes in the electron delocalization and becomes highly polar in the excited state than in the ground state. The Stokes shift has a linear variation with the dipole factor, which is written between square brackets in the expression

$$
v_{a}-v_{f} \approx\left(\frac{(\varepsilon-1)}{(2 \varepsilon+1)}-\frac{\left(n^{2}-1\right)}{\left(2 n^{2}+1\right)}\right) \frac{\left(\mu_{e}-\mu_{g}\right)^{2}}{a^{3} h c}
$$




$$
D_{f}=\frac{(\varepsilon-1)}{(2 \varepsilon+1)}-\frac{\left(n^{2}-1\right)}{\left(2 n^{2}+1\right)},
$$

where $D_{f}$ is dipole factor, $\nu_{a}$ and $\nu_{f}$ are the absorption and fluorescence peaks in wave numbers respectively, $\varepsilon$ is the dielectric constant and $n$ is the solvents refractive index. $\mu_{e}$ is the dipole moment of the solute in the excited state. $\mu_{g}$ is representing the dipole moment of the solute the ground states, respectively, and $a$ is the radius of the solvent cage (which could be around a few hundred nanometers) around the solute.

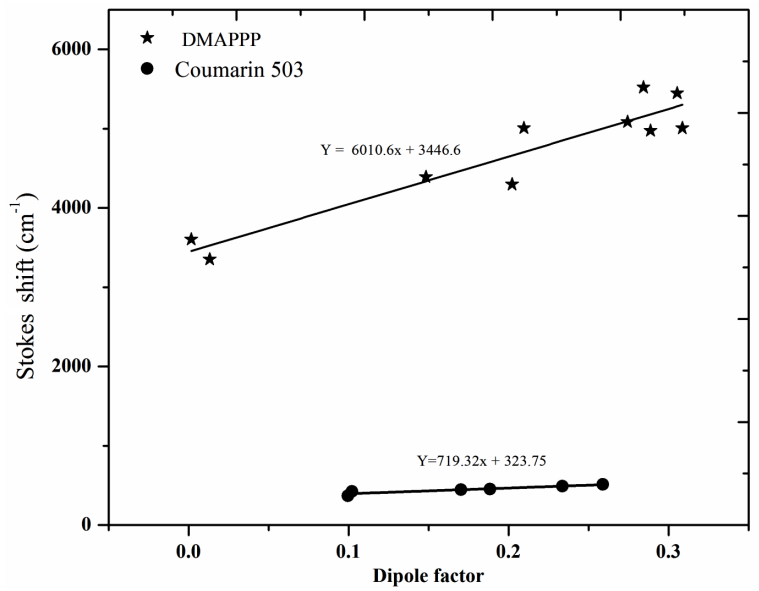

Fig. 2. Variation in the Stokes shift of DMAPPP and coumarin 503 in solution with a dipole factor for different solvents.

Figure 2 shows a plot of the Stokes shift as a function of dipole factor for representative solvents. This dipole factor is a measure of dipole-dipole interaction between the solvents and the solute. One can easily see that DMAPPP is more polar than coumarin 503, under same identical conditions. This result indicates that all these dyes exhibit large changes in the dipole moment in the excited state, but for DMAPPP, the change is much greater than for coumarin 503, as the slope is about eight times greater for the former.

\subsubsection{Quantum yield of fluorescence}

The fluorescence quantum yields $\left(\Phi_{F}\right)$ of DMAPPP and coumarin 503 were measured using diluted solutions. The concentration was kept at $0.65 \mathrm{mM}$ for each solution. The quantum yield can be measured using the following equation [23]:

$$
\Phi_{f}(s)=\Phi_{f}(R) \frac{A_{R} n_{S}^{2} \int I_{S}(\bar{\nu}) \mathrm{d} \nu}{A_{S} n_{R}^{2} \int I_{R}(\bar{\nu}) \mathrm{d} \nu},
$$

where the indices $S$ and $R$ refer to the sample and reference, respectively, and the integral over $I$ represent the area under the fluorescence spectrum. $A$ is the optical density, and $n$ is the refractive index of the solvents.

Tables I and II show the quantum yields of fluorescence $\left(\Phi_{F}\right)$ for DMAPPP and coumarin 503. It can be seen that the $\Phi_{F}$ of DMAPPP is higher than that of coumarin 503 under identical conditions.

\subsection{Amplified spontaneous emission}

To study the ASE properties of DMAPPP under high power excitation, DMAPPP was dissolved in acetone at a concentration of $2 \mathrm{mM}$. This solution was transversely excited with a UV laser. At a pump power of $9 \mathrm{~mJ}$, the ASE spectrum was recorded at $532 \mathrm{~nm}$ with a narrow spectral bandwidth $6 \mathrm{~nm}$ (FWHM) as shown in Fig. 3. This ASE peak is coinciding with the maximum of the fluorescence emission spectrum at this concentration. When the concentration increased to $6 \mathrm{mM}$, there was no significant red shift observed.

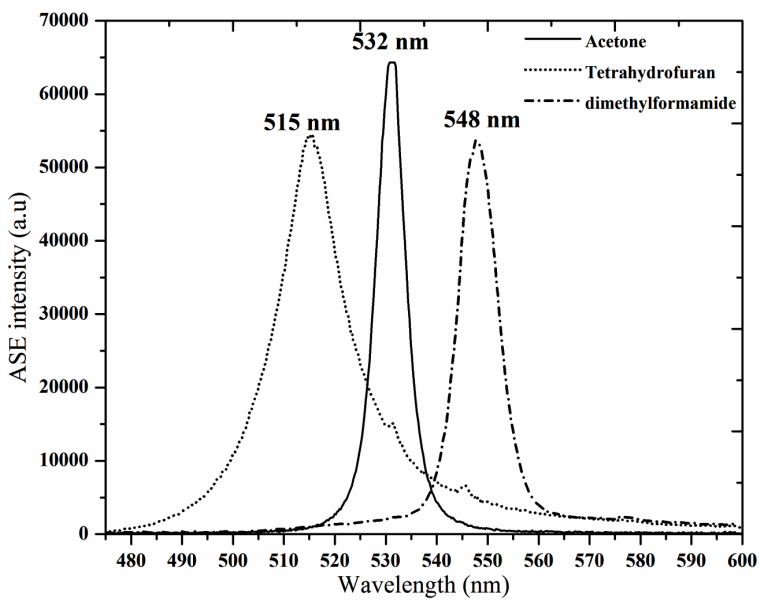

Fig. 3. ASE spectra of DMAPPP in acetone, tetrahydrofuran, and DMF at a concentration of $2 \mathrm{mM}$.

The ASE efficiency of DMAPPP in acetone was measured using gen-tec energy meter. The input energy was $9 \mathrm{~mJ}$ (measured after the cylindrical lens). The sample was excited transversely by focusing laser energy as a strip of light on cuvette. ASE was emanating as a cone of intense light on the both sides of the cuvette perpendicular to laser excitation. The energy of ASE was measured and it was found to be $2.8 \mathrm{~mJ}$. Hence the efficiency was $31 \%$. In a similar fashion, ASE efficiency of DMAPPP was measured in different solvents. Table I shows that the ASE efficiency depends on the solvent environments.

Table I shows the ASE spectra of DMAPPP in different solvents having different polarities under identical conditions; the concentration and the pump power energy were kept at $2 \mathrm{mM}$ and $9 \mathrm{~mJ}$, respectively. In general, it was found that when the dielectric constant increased, the emission wavelength increasingly red shifted (see Fig. 3). In addition, the ASE in acetic acid, methanol, benzene, and toluene was not detected even at high pump power energy and concentration. In toluene and benzene this may be due to the low solubility of the DMAPPP for these solvents. The absence of the ASE spectra in acetic acid may be due to the protonation of N-dimethylamino group of DMAPPP with responsible for their photo properties. Here the methanol plays a similar role by deactivation of the lone pair of N-dimethylamino group by hydrogen bonding; this slightly appears in ethanol which gives 
poor ASE. These results were compared with coumarin 503 as standated material (Table II).

\section{TABLE II}

The dielectric constant $\varepsilon$, spectral and ASE properties: $\lambda_{\max }[\mathrm{nm}]$ for absorption (A), fluorescence (F) and ASE; $\Phi_{F}$; ASE efficiency $\eta[\%]$ of coumarin 503 in different solvents.

\begin{tabular}{l|c|c|c|c|c|c}
\hline \hline \multirow{2}{*}{\multicolumn{1}{c|}{ Solvent }} & \multirow{2}{*}{$\varepsilon$} & \multicolumn{3}{|c|}{$\lambda_{\max }$} & \multirow{2}{*}{$\Phi_{F}$} & \multirow{2}{*}{$\eta$} \\
\cline { 3 - 6 } & 2.27 & 377 & 449 & 459 & 0.25 & 9 \\
\hline benzene & 2.38 & 378 & 455 & 465 & 0.23 & 7 \\
toluene & 4.81 & 381 & 459 & 490 & 0.44 & 5 \\
chloroform & 6.15 & 482 & 465 & - & 0.11 & 0 \\
acetic acid & 7.58 & 384 & 476 & 485 & 0.40 & 11 \\
tetrahydrofuran & 20.7 & 389 & 489 & 495 & 0.52 & 13 \\
acetone & 24.5 & 393 & 492 & 503 & 0.73 & 16 \\
ethanol & 32.7 & 395 & 492 & 500 & 0.40 & 9.7 \\
methanol & 36.7 & 398 & 495 & 500 & 0.31 & 6 \\
dimethylformamide & 37.5 & 398 & 496 & 501 & 0.27 & 5 \\
acetonitrile & & & & &
\end{tabular}

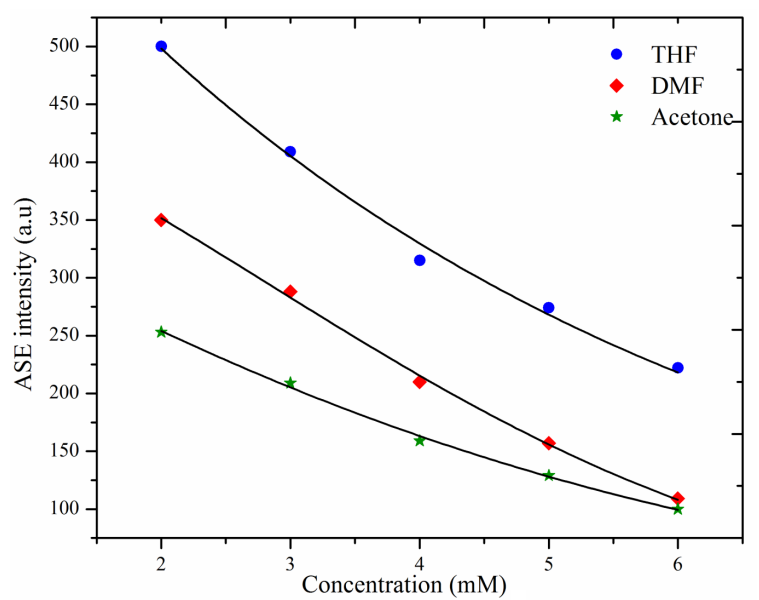

Fig. 4. ASE intensities of DMAPPP in DMF, acetone, and THF as a function of a concentration.

Figure 4 shows the variation in the ASE intensities of DMAPPP as a function of the concentration. The concentration was taken from 2 to $6 \mathrm{mM}$. The solvents were acetone, dimethylformamide (DMF), and tetrahydrofuran (THF). The pump power was $9 \mathrm{~mJ}$. It was found that, as the concentration increased, the intensity of the ASE decreased for each solution.

Figure 5 shows the comparison of the ASE intensity of DMAPPP dissolved in different solvents DMF, acetone and THF under identical condition. These solvents have been chosen because they give high ASE intensities. Moreover, they represent high medium and low dielectric constants. The concentration was kept at $2 \mathrm{mM}$ for each solution; and the pump power was taken from 3 to $15 \mathrm{~mJ}$. It was found that as the pump power was increased, the intensity of DMAPPP in acetone increased slowly, while in THF the intensity increased rapidly.

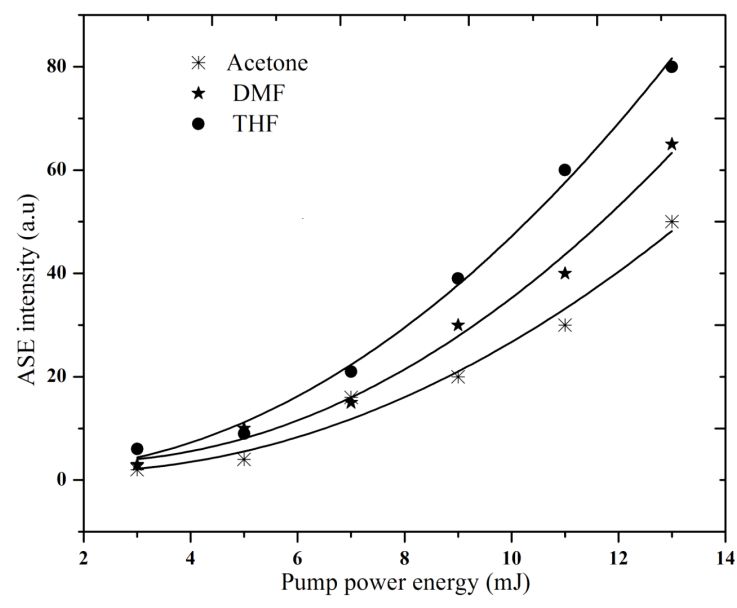

Fig. 5. ASE intensities of DMAPPP in DMF, acetone, and tetrahydrofuran (THF) as a function of pulse energy at a pump power energy.

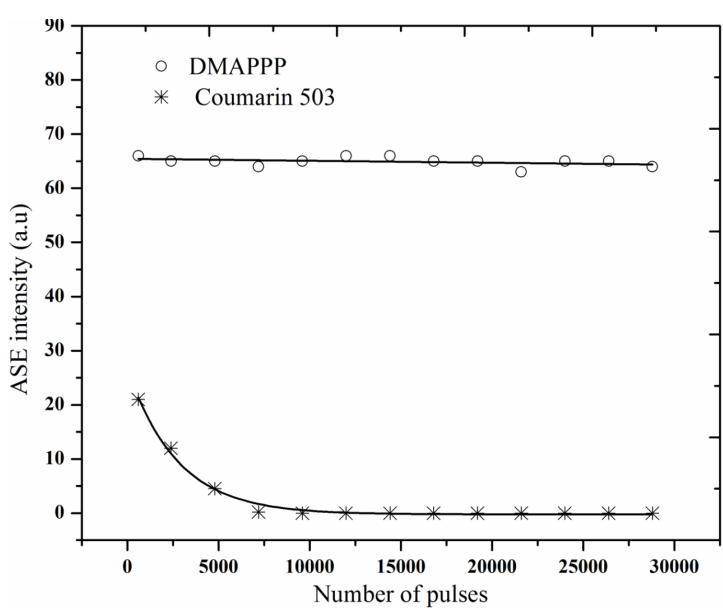

Fig. 6. The photochemical stability of DMAPPP in DMF and coumarin 503 in ethanol

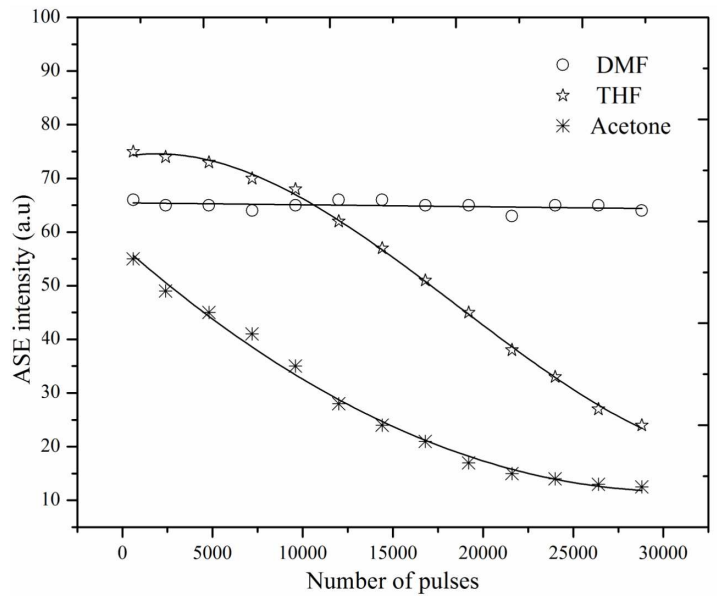

Fig. 7. The photochemical stability of DMAPPP in three different solvents at a concentration of $2 \mathrm{mM}$. 


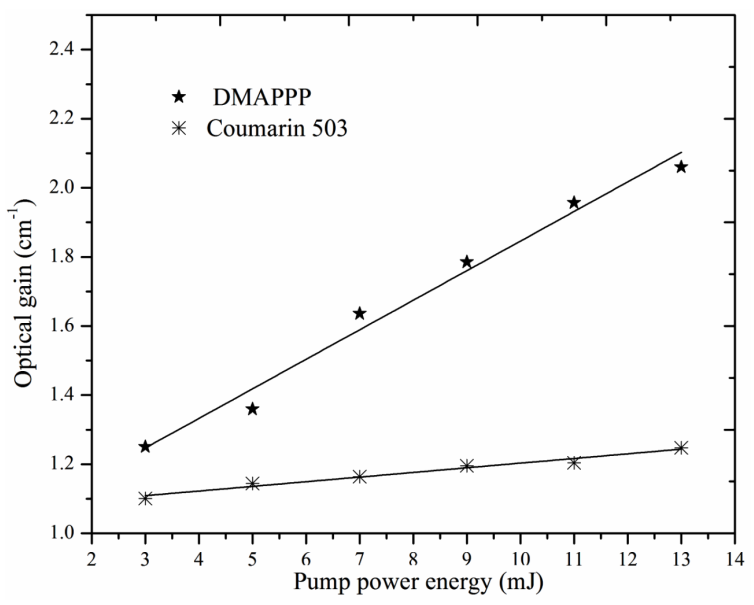

Fig. 8. The relationship between the optical gains vs. pump pulse energy for DMAPPP in DMF and coumarin 503 in ethanol at a concentration of $0.6 \mathrm{mM}$.

The photochemical stability of the DMAPPP in DMF at a concentration of $2 \mathrm{mM}$ was compared to that of $\mathrm{C}$ 503 in ethanol. These solutions were pumped by UV laser of $\mathrm{Nd}$ :YAG with pulse energy of $9 \mathrm{~mJ}$ and a repetition rate of $10 \mathrm{~Hz}$. After 30000 pulses, the ASE intensity of DMAPPP remained constant, whereas that of C 503 dropped to $50 \%$ of the initial intensity after 3000 pulses, and disappeared completely at 7000 pulses as shown in Fig. 6.

The photochemical stability of DMAPPP in different solvents was studied under the same operating conditions. After 30000 pulses, the ASE intensity of THF and acetone was dropped to $32 \%$ and $25 \%$, respectively as seen in Fig. 7 .

The optical gain of DMAPPP in DMF was measured for the concentration $2 \mathrm{mM}$. Coumarin 503 in ethanol was prepared. The pump pulse energy was varied from $3 \mathrm{~mJ}$ to $15 \mathrm{~mJ}$; the excitation wavelength was $355 \mathrm{~nm}$. The ASE intensity was measured for two lengths of excitation $l_{1}=0.5 \mathrm{~cm}$ and $l_{2}=0.3 \mathrm{~cm}[26,27]$ :

$$
\frac{I_{1}}{I_{2}}=\frac{\mathrm{e}^{\gamma l_{1}}-1}{\mathrm{e}^{\gamma l_{2}}-1},
$$

where $l$ is the length of excitation and $\gamma$ is the optical gain.

The results show that the optical gain of DMAPPP in DMF is higher than the coumarin 503 in ethanol as shown in Fig. 8.

\section{Conclusion}

The spectral and ASE behaviors of DMAPPP and coumarin 503 were studied under the influence of concentrations, solvent environments, and laser power excitation levels. Quantum yield, the Stokes shift and ASE were studied. DMAPPP showed large the Stokes shift, and showed also a strong brightness, and green ASE. The most important features of DMAPPP are: (1) the ASE intensity and the photochemical stability of DMAPPP were remarkably high compared with coumarin 503, (2) and the ASE emission from DMAPPP is tunable in the wavelength region between 515 and $548 \mathrm{~nm}$ according to the variety of solvents.

\section{Acknowledgments}

The authors would like to extend their sincere appreciation to the Deanship of Scientific Research at King Saud University for its funding of this research through the Research Group Project No. RGP-223.

\section{References}

[1] A.O. Elzupir, A.E.M. Saeed, I.E. Barakat, J.H. Van der Westhuizen, Int. J. Curr. Pharm. Res. 5, 23 (2013).

[2] A.R. Jagtap, V.S. Satam, R.N. Rajule, V.R. Kanetkar, Dyes Pigm. 91, 20 (2011).

[3] Y.F. Sun, Y.P. Cui, Dyes Pigm. 78, 65 (2008).

[4] Y. Kitaoka, T. Sasaki, S. Nakai, A. Yokotani, Y. Goto, M. Nakayama, Appl. Phys. Lett. 56, 2074 (1990).

[5] J. Indira, P.P. Karat, B.K. Sarojini, J. Cryst. Growth 242, 209 (2002).

[6] S.J. Sun, G. Schwarz, R.H. Kricheldorf, T.C. Chang, J. Polym. Sci. A Polym. Chem. 37, 1125 (1999).

[7] K. Krohn, K. Steingrover, M. Srinivasa Rao, Photochemistry 61, 931 (2002).

[8] Y. Sato, M. Morimoto, H. Segawa, T. Shimidzu, J. Phys. Chem. 99, 35 (1995).

[9] A.O. Doroshenko, A.V. Grigorovich, E.A. Posokhov, V.G. Oivovarenko, A.P. Demchenko, Mol. Eng. 8, 199 (1999).

[10] K. Rurack, J.L. Bricks, G. Reck, R. Radeglia, U. Resch-Genger, J. Phys. Chem. A 104, 3087 (2000).

[11] N. Marcotte, S. Fery-Forgues, D. Lavabre, S. Marguet, V.G. Pivovarenko, J. Phys. Chem. A 103, 3163 (1999).

[12] P.K. Das, R. Pramanik, D. Banerjee, S. Bagchi, Spectrochim. Acta A 56, 2763 (2000).

[13] Z. Xu, G. Bai, C. Dong, Spectrochim. Acta A 62, 987 (2005).

[14] P. Wang, S. Wu, J. Photochem. Photobiol. A Chem. 86, 109 (1995).

[15] N. DiCesare, J.R. Lakowicz, Anal. Biochem. 301 , 111 (2002).

[16] Y. Wang, J. Phys. Chem. 89, 3799 (1985).

[17] Y.B. Jiang, X.J. Wang, L. Lin, J. Phys. Chem. 98, 12367 (1994)

[18] S.A. El-Daly, M. Gaber, S.S. Al-Shihry, Y.S. El Sayed, J. Photochem. Photobiol. A Chem. 195, 89 (2008).

[19] K.H. Ibnaouf, M.K.M. Ali, A.O. Elzupir, M.A. Ibrahem, H. Idriss, A.S. Alaamer, M.A. Alrajhi, M.S. Alsalhi, Digest J. Nanomater. Biostruct. 12, 4322017.

[20] S. Prasad, K.H. Ibnaouf, M.S. AlSalhi, V. Masilamani, 55, 727 (2014).

[21] E.Z. Lippert, Naturforschung 10, 541 (1955).

[22] N. Mataga, Y. Kaifu, M. Koizumi, Bull. Chem. Soc. Jpn. 29, 465 (1956).

[23] K.H. Ibnaouf, S. Prasad, M.S. AlSalhi, A. Hamdan, M.B. Zaman, L. El Mir, J. Lumin. 149, 369 (2014). 